\title{
Une conférence sur la croissance de formes, en hommage à Yves Couder
}

Compte rendu de Benoît Roman (benoit@pmmh.espci.fr)

Laboratoire PMMH, ESPCI, 10 rue Vauquelin, 75231 Paris Cedex 05

Dans les dernières décennies,

la physique non linéaire et

hors d'équilibre a généré

avec succès des concepts

puissants liés aux instabilités,

à la formation de motifs

et au chaos spatio-temporel.

Ces concepts et méthodes

sont maintenant largement

utilisés dans de nombreux

domaines, allant de la physique

de la matière condensée à la

géophysique et à la biologie.

La conférence "New trends

in growth and form" avait

pour but d'éclairer les récents

développements

et les nouvelles directions de

recherche de cette physique.

(1) Phyllotaxie : arrangement spiralé que l'on retrouve, par exemple,

pour les écailles de pommes de pin.

La conférence a été organisée avec l'aide du GDR MéPhy, de la fédération " Dynamique des systèmes complexes », du CEA, de la fondation Pierre-Gilles de Gennes, du laboratoire Matière et Systèmes complexes (UP7), de l'Université de Provence, et du CNRS. Pour plus d'informations, on pourra consulter le site web de la conférence à l'adresse :

www.pmmh.espci.fr/ mephy/ogaf/index.php
La rencontre "New trends in growth and form" a été organisée à Agay (Var), du 20 au 25 juin 2010 : elle a réuni pendant une semaine plus d'une centaine de participants venant d'Europe, mais aussi des USA, d'Isräl, du Japon et du Canada. Parmi ceux-ci se trouvaient des experts internationaux de grand renom (J. Bush, J. Fineberg, J. Gollub, L. Kadanoff, A. Libchaber, I. Procaccia, B. Shraiman, H. Swinney) qu'Yves Couder a côtoyés. Un dialogue s'est ainsi établi entre des chercheurs issus de disciplines différentes (de la mécanique des fluides à la biologie végétale et animale, en passant par la mécanique des solides et la physique statistique). Pendant cette semaine, les participants ont pu partager une approche commune : l'intérêt pour les expériences " de coin de table ", comme point de départ d'une réflexion approfondie sur l'organisation et l'émergence de formes.

Yves Couder joue un rôle majeur dans plusieurs domaines de la physique : turbulence, instabilités, singularités, mécanique de la morphogénèse végétale (nervation des feuilles, phyllotaxie $\left.\mathrm{e}^{(1)}\right)$, écoulements interfaciaux (interaction goutte-onde et analogie avec la mécanique quantique). Ses expériences très astucieuses ont inspiré et ouvert la voie à beaucoup d'autres chercheurs, tant en France qu'à l'étranger. Il a ainsi reçu le Prix de l'IFP (Académie des sciences, 1993), le Prix Gentner-Kastler (Deutsche Physikalische Gesellschaft et Société Française de Physique, 2007) et le Prix des trois physiciens (2007).

On note aussi l'attachement d'Yves Couder à l'enseignement expérimental, en prise directe avec la recherche, dont il fut un des promoteurs à l'université Paris Diderot. Plusieurs de ses sujets de recherche les plus imaginatifs ont ainsi démarré dans le cadre du programme "Phy Ex », où les étudiants se voient confier un sujet expérimental nouveau (dont ils doivent monter une petite expérience).

Plus qu'un hommage formel, il s'agissait ici d'une véritable conférence de recherche, une occasion de susciter interactions et collaborations scientifiques, en présentant dans une même rencontre des travaux actuels dans plusieurs thématiques abordées par Yves Couder, dont voici quelques exemples.

\section{Motifs et physique non linéaire}

Dans ce volet de la conférence, le dénominateur commun de toutes les présentations était une filiation avec la physique non linéaire, à l'essor de laquelle Yves Couder a beaucoup contribué. Dans ce domaine, les physiciens étudient le comportement non linéaire (instabilités, motifs et évolutions) de systèmes divers : mécanique des fluides, matériaux granulaires, mécanique des solides, plasmas... Au-delà de la spécificité de chaque système, un langage commun permet d'unifier et de classifier les comportements en des classes universelles.

La conférence d'Agay a, dans le même esprit, réuni des contributions dans des domaines très divers, mais en s'attachant à y retrouver des caractéristiques communes. Un de ces systèmes non linéaires modèles, dont l'étude est toujours active (plusieurs présentations lors de la conférence), est la digitation visqueuse (instabilité de SaffmanTaylor) qui apparaît quand on repousse un fluide avec un autre fluide moins visqueux. Des études de la formation de stalactites de glace ou de motifs de convection planétaire ont été présentées. 


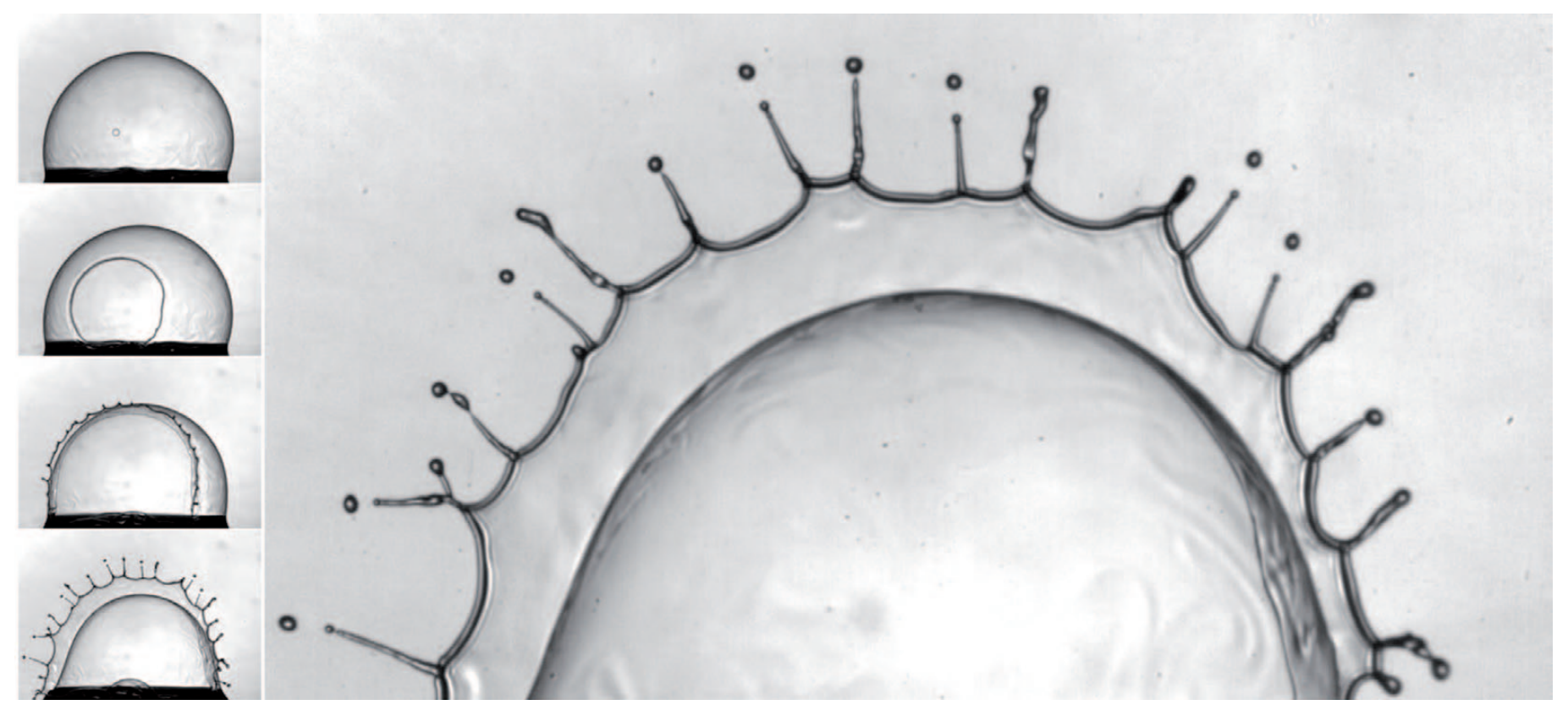

1. Éclatement d'une bulle d'eau. Séquence d'images à gauche : stades initiaux de l'éclatement d'une bulle d'eau, remplie initialement d'air en surpression (à cause de la tension superficielle). Le trou apparaît au pied de la bulle, près de son équateur (image du haut) ; il grandit en restant circulaire au départ, tandis que le reste de la bulle se vide. II se forme alors une corolle rattachée au reste de la bulle par un pli (marqué par la ligne interne) ; cette corolle se déstabilise pour former des ligaments liquides, qui se fragmentent à leur tour en gouttes éjectées dans l'atmosphère. Le rayon initial de la bulle est de $10 \mathrm{~mm}$ et l'intervalle entre deux images de la séquence de 2 ms. Image de droite : vue de détail de la déstabilisation de la corolle. (Document : H. Lhuissier et E. Villermaux, IRPHE, Marseille).

\section{Dynamique des interfaces fluides}

Les travaux les plus récents d'Yves Couder concernent la dynamique de gouttelettes rebondissant sur un liquide, et l'interaction avec les ondes produites par l'impact. En particulier, ces expériences ont mis en évidence une belle et surprenante analogie avec la dualité onde-particule de la mécanique quantique [1]. De nombreuses présentations ont également concerné la dynamique d'interfaces fluides : rebonds de gouttes, singularités de détachement d'un jet, motifs de déferlement de vagues, motifs de Faraday localisés, motifs de type phyllotaxie $^{(1)}$, de bulles émergeant à la surface d'un liquide, atomisation de nappes liquides ou de bulles (fig. 1).

\section{Biologie et physique macroscopique}

Cette partie de la conférence a permis de regrouper des travaux qui ont été abordés dans des systèmes très différents, allant de la botanique à la biologie animale, et de faire ressortir les questions de physique communes qu'ils suscitent.

Des questions naturelles (mais pas simples) de physique en biologie concernent la locomotion de micro-organismes : quelle efficacité de propulsion ? Comment la locomotion est-elle orientée par la perception chimique (chimiotaxie, voir glossaire p. 6) ? Le séchage de structures végétales induit des rétrécissements anisotropes et inhomogènes, et donne ainsi lieu à des changements de formes : peut-on comprendre l'ouverture des écailles de pommes de pin, ou l'ouverture en hélice des coques enveloppant certaines graines ?

Mais la biologie s'intéresse aussi à la morphogénèse à l'échelle des tissus. Ces milieux continus en croissance posent des questions nouvelles aux physiciens. En effet, si la croissance du tissu n'est pas homogène, il apparaît des incompatibilités de taille entre ses différentes parties. Ces frustrations peuvent-elles occasionner des instabilités morphogénétiques ? C'est le cas des tumeurs, dont la croissance anarchique peut donner lieu à des corrugations par flambage de la surface (c'est un signe d'une mauvaise évolution des grains de beauté). Les contraintes internes peuvent-elles être à l'origine des veines? (la question se pose pour les méduses, aussi bien que pour les nervures des feuilles végétales). Ces instabilités sont-elles la voie qui permet de passer de l'information génétique à la forme des êtres vivants ? Dans les plantes, des travaux récents (collaboration incluant en particulier Y. Couder - voir encadré, p. 13) ont montré que la croissance anisotrope se faisait en fonction de l'orientation des contraintes. Or, c'est cette croissance anisotrope qui donne à la tige une forme allongée au lieu d'une forme de sphéroïde.

Si la croissance est susceptible de produire des contraintes internes, existe-t-il un processus de régulation (feed-back) basé sur une sensibilité à ces contraintes ? C'est ce que l'on observe sur la croissance périphérique (secondaire) des tiges et des troncs, ce qui leur permet de s'adapter aux sollicitations du milieu (vent). Cette perception des contraintes permettrait aussi de réguler la croissance pour atteindre une certaine taille (embryon de drosophile).

Quelle est la nature de la perception biologique des contraintes au niveau cellulaire ? Les mécanismes proposés pour les cellules animales different de ceux qui concernent les cellules végétales. Ces dernières ont en effet la particularité d'être entourées d'une paroi solide, qui leur confère un comportement mécanique particulier. Se pose également la question de la mesure et de l'estimation de ces contraintes. 


\section{Isotropie et anisotropie de la croissance des plantes : un exemple de sujet évoqué à la conférence}

La colonisation de la terre ferme par les plantes dépend en partie de leur capacité à construire des tiges qui s'élancent vers les airs. Pour ce faire, leur paroi cellulaire est renforcée par des fibres de cellulose (microfibrilles), qui sont synthétisées dans une direction qui dépend des stimuli internes et externes auxquels sont soumises les cellules. Ces fibres forment un corset qui semble guider la croissance anisotrope, maintenant ainsi la forme allongée des tiges.

Que se passe-t-il quand l'orientation des microfibrilles est perturbée par un traitement pharmacologique? D'une part, les sommets des tiges croissent en forme de boule, traduisant la perte de l'anisotropie de croissance. D'autre part, la géométrie du réseau formé par les cellules évolue, comme on le voit sur la figure, vers celle d'une mousse de savon, qui est par essence isotrope. Ainsi, I'anisotropie induite par les microfibrilles est un élément essentiel dans la morphogenèse et l'architecture végétale [2].

\section{Rupture, froissement et physique statistique}

Y. Couder s'est également intéressé aux motifs de rupture et aux singularités de froissement d'une plaque mince. Plusieurs présentations portaient sur ces sujets. Comment se propage un front de fissure dynamique et quelles instabilités subit-il ? Lorsque le milieu présente des inhomogénéités, le front de fissure est déformé et l'étude de sa rugosité doit être traitée statistiquement. De même, les outils de physique statistique sont utilisés pour décrire les arrangements (et un ordre " nématique ") des plis dans des boules de papier froissé, l'organisation de séquences codantes dans un brin d'ADN, ou une transition vers l'irréversibilité dans les écoulements d'une suspension à faible nombre de Reynolds.

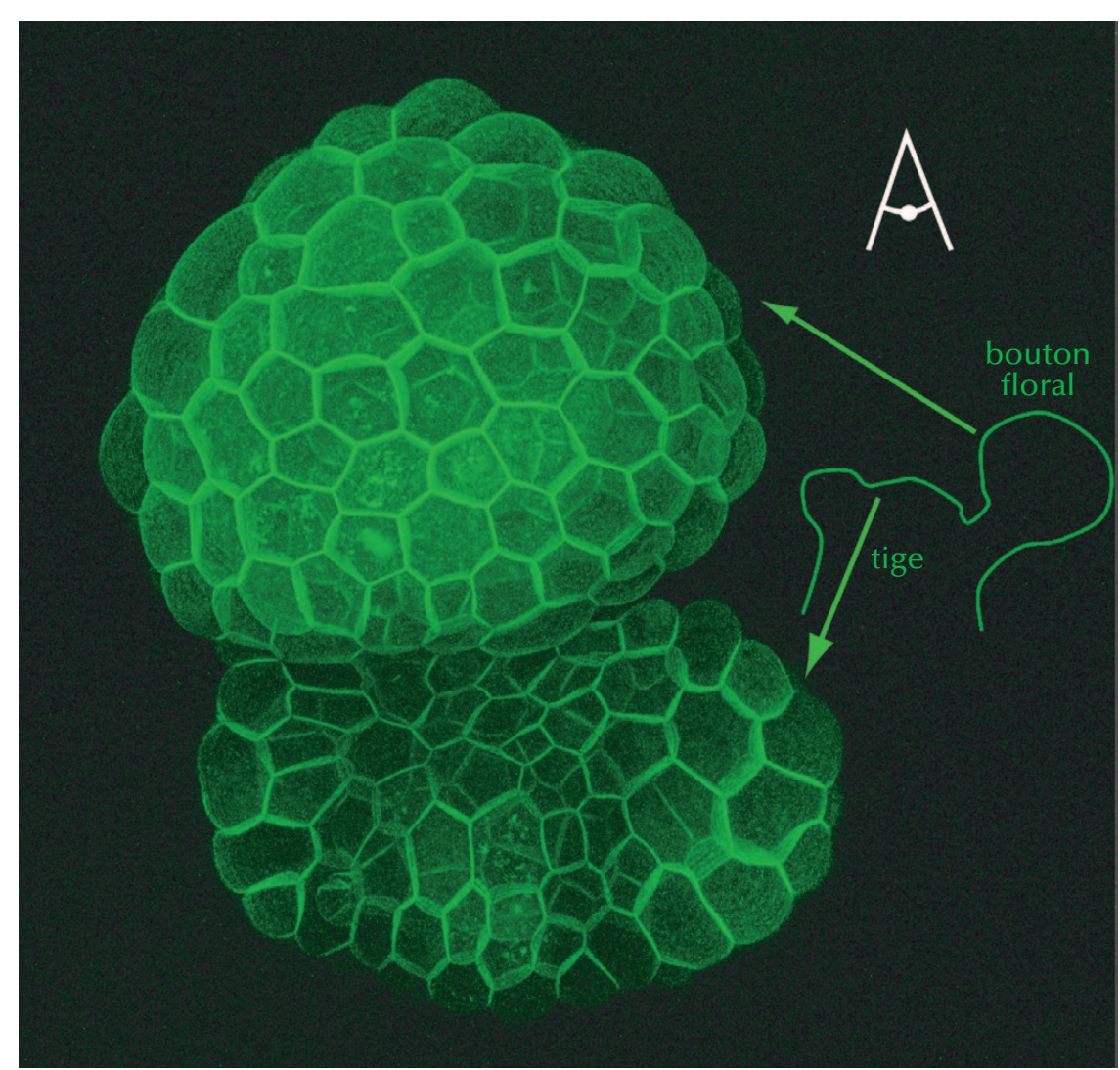

Vue de dessus, par microscopie confocale, de l'extrémité d'une tige portant un bouton floral sur le côté (largeur de l'image : 250 microns; schéma de droite : vue de côté). À la suite d'un traitement rendant la croissance isotrope, les cellules, en général allongées, acquièrent ici une géométrie de bulles de savon, et le bouton floral (en haut) prend une forme quasi sphérique.

(Document : Olivier Hamant, RDP, INRA / CNRS / UCBL / ENS Lyon).

\section{Références}

1• Y. Couder, A. Boudaoud, S. Protière et E. Fort, « Les gouttes marcheuses - Une forme de dualité onde-particule à échelle macroscopique? ", Reflets de la physique, $n^{\circ} 5$ (2007) 20-24.
2• F. Corson, 0. Hamant, S. Bohn, J. Traas, A. Boudaoud et Y. Couder, "Turning a plant tissue into a living cell froth through isotropic growth", Proc. Natl. Acad. Sci. USA 106 (2009) 8453-8458. 